\title{
Systèmes de production locaux et gestion des ressources pastorales en Albanie. Entre stratégies d'acteurs et évolutions institutionnelles
}

\author{
Florjan Bombaj ${ }^{1,3, *}$, Dominique Barjolle ${ }^{2,3}$, Jean-Marc Touzard ${ }^{3}$, François Casabianca ${ }^{4}$ \\ et Simon Gontard ${ }^{5}$ \\ ${ }^{1}$ Université méditerranéenne d'Albanie, Bulevardi Gjergj Fishta 52, Tirana 1023, Albanie \\ 2 ETH Zürich, Institute for Agricultural Sciences, TAN F1, Tannenstrasse 1, 8092 Zürich, Suisse \\ ${ }^{3}$ INNOVATION, Univ Montpellier, CIRAD, INRAE, Institut Agro, Montpellier, France \\ ${ }^{4}$ INRAE, LRDE, Quartier Grossetti, 20250 Corte, France \\ 5 AgroParisTech, 16 rue Claude Bernard, F-75231 Paris, France
}

\begin{abstract}
Résumé - L'article analyse la dynamique des systèmes d'élevage d'un territoire montagneux albanais sous fortes contraintes socio-économiques, géographiques et historiques. L'objectif est de comprendre les processus qui ont conduit à la coexistence de systèmes d'élevage contrastés dans ce territoire et de montrer comment cette coexistence est liée à l'utilisation des ressources pastorales. Suite à la chute du régime communiste en 1991, la forte migration de la population vers les zones de plaine et la croissance de la demande pour les produits laitiers et carnés ont entraîné une augmentation de la production et de la taille des troupeaux dans ce territoire, avec une pression accrue sur les ressources pastorales. Les enquêtes réalisées dans cinq villages permettent de proposer une typologie des systèmes d'élevage, montrant la diversité des résultats économiques et des usages des ressources pastorales. Ces résultats questionnent l'évolution de l'élevage pastoral en Albanie dans le cadre d'une possible intégration de ce pays à l'Union européenne.
\end{abstract}

Mots clés : Systèmes d'élevages / Albanie / produits pastoraux

\begin{abstract}
Local production systems and pastoral resources management in Albania. Between stakeholders strategies and institutional developments. The article analyses the dynamics of livestock systems in an Albanian mountainous territory under strong socio-economic, geographic and historical constraints. The objective is to understand the processes that have led to the coexistence of contrasting livestock systems in this territory and to show how this coexistence is linked to the use of pastoral resources. Following the fall of the communist regime in 1991, the strong migration of the population to the lowland areas and the increase of the demand for dairy and meat products has resulted in increased production and herd size in this territory, with increased pressure on pastoral resources. The surveys carried out in five villages make it possible to propose a typology of livestock systems, showing the diversity of economic results and uses of pastoral resources. These results question the evolution of pastoral farming in Albania in the context of a possible integration of this country into the European Union.
\end{abstract}

Keywords: Livestock systems / Albania / pastoral products

\section{Introduction}

L'Albanie est un petit pays des Balkans se préparant à adhérer à l'Union européenne (UE). L'effondrement du régime communiste en 1991, une libéralisation chaotique et la lenteur

\footnotetext{
$\overline{\text { *Auteur de correspondance }}$ : florjan.bombaj@supagro.fr
}

des nouvelles réformes structurelles ont entraîné une forte instabilité politique, économique et sociale jusqu'au début des années 2000. Pendant cette période, l'agriculture, très vite privatisée, s'est repliée sur une multitude de micro-exploitations paysannes à faible productivité du travail, pendant que l'État conservait partiellement la propriété des terres de pâturages et de forêts (Bourbouze et François, 2001; Bernard et Lerin, 2017). Reconnu en 2000 comme candidat potentiel à 
l'UE, le pays s'est engagé dans la perspective de cette intégration, ce qui demande de poursuivre les réformes économiques et politiques et d'harmoniser sa législation avec celle de l'UE. Des études sont ainsi menées pour identifier les problèmes liés à l'intégration européenne de son agriculture et fournir des recommandations pour réussir ce processus (European Commission, 2019). Cet article veut contribuer à cette réflexion en concentrant l'analyse sur le secteur de l'élevage dans les régions montagneuses, un secteur qui risque d'être très exposé à la concurrence, mais pourrait aussi bénéficier de soutiens dans le cadre de la politique européenne de développement rural.

L'Albanie est en effet un pays montagneux : $45 \%$ de sa surface est située à une altitude supérieure à $1000 \mathrm{~m}$ et plus de la moitié des terres agricoles ont une pente supérieure à $5 \%$. Dans les zones de montagne, la mécanisation de l'agriculture est difficile, les processus d'érosion des sols sont importants et les rendements des cultures inférieurs à ceux des plaines (Dhimitri et al., 2012). Les élevages bovins, ovins et caprins, à la fois laitiers et à viande, y fournissent l'essentiel de la production agricole au niveau national (INSTAT, 2019) et dépendent largement de l'utilisation de pâturages. Durant la période communiste, ces pâturages appartenaient à l'État, étaient gérés au niveau central et avaient été accordés en priorité aux coopératives d'élevage créées après 1953. Depuis la fin du régime communiste en 1991, la transition politique et économique a bouleversé les systèmes d'élevage et les droits de propriété et d'usage des pâturages (Müller et Munroe, 2008).

Dans un contexte de changements réglementaires fréquents, des pâturages étatiques, privés et communaux coexistent, offrant de nouvelles contraintes ou opportunités aux éleveurs. Certains éleveurs ont ainsi augmenté leur cheptel, entraînant une pression accrue pour l'utilisation des pâturages (Bombaj et al., 2018). Les changements dans la gestion des ressources naturelles semblent avoir favorisé une différenciation des stratégies des éleveurs appelant à revoir leur coordination à l'échelle locale. L'article analyse la diversité de ces stratégies dans un territoire de montagne d'Albanie au regard des différents modes de gestion des pâturages, offrant une contribution pour comprendre l'évolution du secteur de l'élevage de montagne et sa capacité à faire face à l'intégration européenne.

Dans un premier temps, nous présentons le cadre d'analyse qui associe systèmes d'élevage et gestion des ressources pastorales vues comme des biens communs (Sect. 2). Nous précisons ensuite la région étudiée et la méthode, fondée sur des enquêtes dans cinq villages (Sect. 3). Les résultats sont ensuite exposés, montrant les liens entre les différents types de systèmes d'élevage, leurs résultats économiques et les modes d'accès au pâturage (Sect. 4). Ces résultats sont enfin discutés en réinterrogeant les perspectives d'évolution de l'élevage en montagne dans le cadre d'une possible intégration européenne (Sect. 5).

\section{Cadre théorique et question de recherche}

Pour comprendre la dynamique actuelle de l'élevage, et celle d'occupation et d'utilisation des ressources pastorales, il est important d'analyser la diversité, la coexistence et l'évolution des systèmes de production, d'élevage et d'activités dans un territoire, ici une région montagneuse et marginalisée (Manoli et al., 2011). Un système de production correspond à un ensemble d'exploitations ayant accès à des ressources comparables (même gamme de troupeaux, niveau d'équipement, utilisation foncière ou travail disponible), placées dans des conditions socio-économiques similaires et pratiquant une combinaison de productions donnée (Cochet et al., 2007). Le système d'élevage est une composante du système de production, qui se définit à l'échelle du troupeau, et est constitué d'un ensemble d'éléments organisés par l'éleveur afin de valoriser les ressources au moyen d'animaux domestiques pour obtenir diverses productions ou pour répondre à d'autres objectifs (Landais, 1992). Le système d'activités comprend le système de production et les activités et sources de revenu complémentaires des unités familiales impliquées dans l'activité agricole et d'élevage: double activité, rente ou mise à disposition de capitaux tels que les retraites ou des transferts liés à l'émigration. La notion de système d'activité est très importante dans le contexte étudié, car il permet de comprendre pourquoi certaines exploitations se maintiennent alors que les conditions de leur rentabilité économique ne sont pas remplies (Biba et Pluvinage, 2006).

La compréhension des dynamiques d'élevage et d'usage des pâturages appelle aussi à prendre en compte les effets des institutions et des politiques publiques, jouant sur les règles d'usage des ressources pastorales. La littérature sur la décentralisation des politiques publiques (Addison et al., 2013 ; Andersson et Ostrom, 2008) montre ainsi que dans les pays qui ont décentralisé la gestion de leurs ressources naturelles, cette décentralisation ne conduit pas uniformément à une gouvernance locale efficace. De manière générale, la clé de cette efficacité réside dans les relations établies entre les acteurs concernés au sein d'une communauté. Dépassant la recherche de leur intérêt immédiat, ceux-ci peuvent en effet arriver à un mode de «self gouvernance» des ressources efficace et durable, fondé sur des normes, vues comme des «attributs de la communauté», et des règles qui cadrent l'action et établissent les droits. Selon Ostrom (2005), trois catégories de conditions initiales sont nécessaires pour que les ressources naturelles soient gérées collectivement: a) la prise en compte des caractéristiques biophysiques des ressources; b) les caractéristiques socio-économiques de la communauté qui utilise et gère la ressource; c) les dispositifs institutionnels qui comprennent les règles et droits d'usage, et les relations formelles et informelles pouvant favoriser confiance et coopération entre acteurs.

Lorsque, dans une communauté, les niveaux attendus de coopération, d'autonomie et d'autodétermination n'ont pas été atteints, les utilisateurs locaux de la ressource peuvent développer des stratégies individuelles qui la menacent à long terme, tandis que les plans de gestion officiels sont largement ignorés et non appliqués (Addison et al., 2013). Dans ces situations, la réglementation renforcée des biens publics peut alors augmenter la concurrence pour leur accès (Beesley, 1985), ce qui devient intenable lorsque les contextes économiques, politiques et sociaux changent (Huband et al., 2010). Des recherches récentes confirment aussi que les communautés pastorales préfèrent opter pour des pratiques cadrées par des institutions traditionnelles et publiques qui garantissent un accès réciproque aux ressources pastorales 
(Lutta et al., 2020), rejoignant les propositions de «gouvernance polycentrique » suggérée par Ostrom. D'autres recherches enfin montrent les difficultés d'une gestion durable des ressources pastorales à cause des conflits entre les acteurs locaux et de l'arrivée de nouveaux acteurs qui affaiblissent et rendent plus difficile l'action collective locale (Afane et Gagnol, 2014).

L'hypothèse de cet article est qu'après 1991, les systèmes d'élevage se sont progressivement adaptés au nouveau contexte politique et économique. Certains éleveurs ont augmenté leur cheptel, ce qui a entraîné des changements dans la gestion et l'intensité de l'utilisation des pâturages, accentuant la différenciation possible des stratégies de production des éleveurs locaux. Cet article vise à répondre à la question de recherche suivante: comment les ruptures institutionnelles dans les règles de gestion des pâturages ontelles affecté les systèmes de production et permis la coexistence de systèmes d'élevage hétérogènes, aujourd'hui en concurrence pour la ressource pastorale?

\section{Matériel et méthode}

\subsection{Présentation de la région étudiée}

La région de Korçë, située au sud-est de l'Albanie, est traversée par une grande plaine agricole (la deuxième du pays), entourée de chaînes de montagnes dont les sommets atteignent plus de $2000 \mathrm{~m}$ d'altitude. Dans ces massifs, l'élevage extensif de bovins et de petits ruminants s'est développé (Mavromati et al., 2011), profitant de conditions pédoclimatiques favorables à la croissance de l'herbe (précipitations relativement importantes, températures douces en été). Nous avons choisi d'étudier dans cette région la municipalité de Vithkuq, qui s'étend sur une zone agropastorale connue pour ses produits pastoraux de qualité dans toute l'Albanie (Bombaj et al., 2017). Ce territoire concentre aussi une série de conditions et d'enjeux qui en font un cas d'étude pertinent: enclavement, économie spécialisée dans les productions pastorales et sylvicoles, exode, pauvreté rurale, difficultés à reconstruire des cadres collectifs pour gérer l'espace local, diversité a priori de systèmes d'élevage.

Dans cette région, comme dans le reste de l'Albanie, trois périodes principales de gestion des pâturages peuvent être observées au cours de l'histoire. La première période, sous l'Empire ottoman, se caractérise par un régime mixte où coexistaient pâturages d'État, privés et communaux, avec des conditions d'accès basées sur les besoins des communautés locales. La deuxième période, à partir de 1944 sous le régime communiste, est la gestion centralisée de pâturages appartenant à l'État et accordés en priorité aux coopératives d'élevage créées après 1953. La troisième période, dans le contexte postcommuniste à partir de 1991, voit de nouveau un régime mixte où coexistent pâturages étatiques, privés et communaux.

À partir des années 2000, le développement de l'élevage a surtout concerné les ovins, particulièrement adaptés aux conditions locales. Le nombre d'animaux a augmenté, malgré une baisse de la population à l'échelle de la commune (Bombaj et al., 2019). Pour les éleveurs locaux, le cœur du système pastoral reste la zone d'estive du «massif de Rrungaja» (Gontard, 2016). Mais après 28 ans de «période de transition postcommuniste $»$, les stratégies des éleveurs, mais aussi des acteurs institutionnels et de la population locale dans la gestion de ces pâturages sont mal connues. Les dynamiques de conduite au pâturage des troupeaux ont évolué dans le temps et les systèmes d'élevage actuels s'inspirent de ces dynamiques passées (Gontard, 2016). Les systèmes de production en concurrence sur la ressource pastorale ont des pratiques et des résultats économiques hétérogènes. Ils sont animés par des dynamiques différentes: alors que certains décapitalisent, d'autres augmentent sensiblement la taille de leur troupeau.

\subsection{Méthode}

Notre recherche repose sur une collecte de données qualitatives et quantitatives, primaires et secondaires. $\mathrm{Au}$ niveau régional et local, des archives, cartes et registres ont été consultés et quatre-vingt-deux entretiens ont été menés afin d'analyser le fonctionnement des systèmes d'élevage, les logiques d'organisation des filières lait, fromage et viande de la municipalité, et les relations entre les acteurs.

La méthode associe plusieurs approches: le diagnostic agraire qui permet de caractériser le système agraire d'un territoire donné (Cochet, 2011); l'analyse de la performance économique des exploitations (Cochet et al., 2007); la méthode Likert (1932) pour évaluer les liens entre la ressource pastorale, les stratégies d'acteurs et les évolutions institutionnelles.

La méthode a suivi cinq étapes :

- Étape 1-Recherche documentaire et analyse du paysage pour déterminer les principaux villages composant la municipalité de Vithkuq (l'une des plus grandes municipalités de la région de Korçë) qui utilisent la ressource pastorale. Ce travail a combiné une analyse d'images satellites et les résultats d'entretiens préalables avec les autorités régionales, puis avec des membres clés des villages sélectionnés. Cinq villages ont été choisis en tenant compte de l'importance des pâturages, mais aussi du nombre d'éleveurs, de la taille et de la diversité des élevages, des liens avec les laiteries de la région.

- Étape 2-En s'appuyant sur les premiers entretiens mais aussi la base de données du ministère de l'Agriculture par le biais du projet européen «PAZA Project», 33 éleveurs ont ensuite été sélectionnés dans ces villages à partir d'une typologie structurelle qui privilégie la spécialisation et la taille des élevages : éleveurs spécialisés avec généralement des troupeaux importants, éleveurs orientés vers l'autosubsistance, situations intermédiaires associant des élevages mixtes et de taille moyenne. Les systèmes de production et les modes de gestion des pâturages ont été renseignés à partir d'entretiens semi-directifs avec les éleveurs.

- Étape 3-Identification des caractéristiques de chaque système d'élevage en termes de performance économique et d'utilisation de la ressource pastorale. Les résultats ont permis de questionner les différents modes d'allocation de la ressource pastorale en termes d'efficacité économique pour les éleveurs locaux.

- Étape 4-Enquêtes complémentaires sur la dynamique des filières pastorales et les perspectives possibles après les changements gouvernementaux et institutionnels dans l'utilisation des pâturages: laiterie, gestionnaires de pâturage, experts du développement rural... 


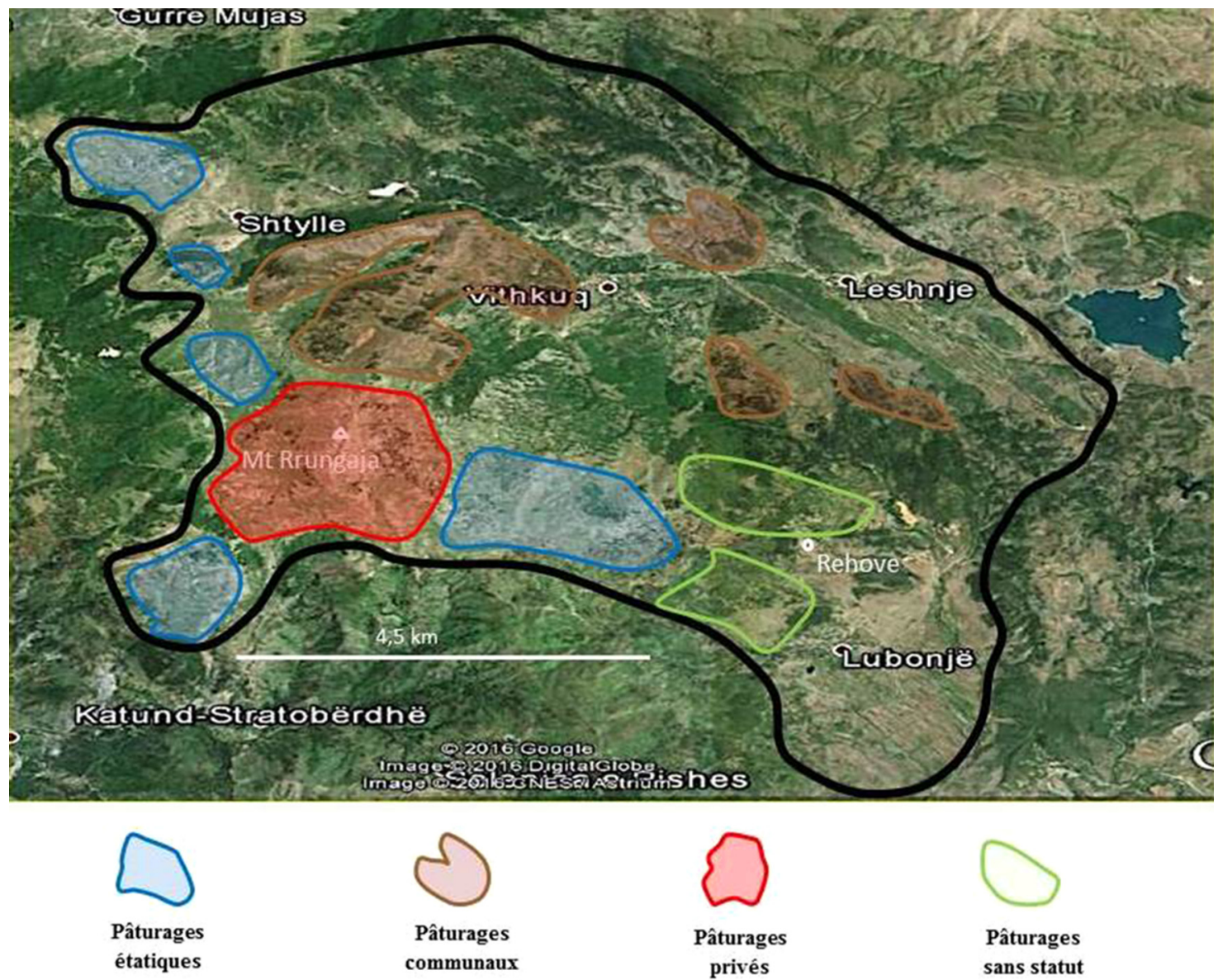

Fig. 1. Zone d'étude (5 villages) et identification territoriale des différents types de gouvernance des pâturages.

Fig. 1. Study area (5 villages) and territorial identification of the different types of pasture governance. (Source: d'après Gontard, 2016; élaboré par les auteurs).

- Étape 5-Analyse des résultats et discussion. En utilisant cette approche, il a été possible d'obtenir une description globale et détaillée des systèmes d'élevages locaux qui utilisent la ressource pastorale et l'identification des systèmes qui sont économiquement les plus performants.

\section{Résultats}

\subsection{Organisation spatiale des utilisateurs de la ressource pastorale}

Le pâturage représente l'alimentation principale des troupeaux de la municipalité de Vithkuq. C'est la formation végétale dominante des milieux ouverts du massif, accueillant des écosystèmes riches et diversifiés. Les systèmes agropastoraux suivent une logique géographique adaptée au milieu montagnard. L'exploitation de cette ressource est un enjeu très fort de la gestion à la fois des élevages et du paysage. Pour les transhumants, le cœur du système est la zone d'estive du massif de Rrungaja (Fig. 1). Les pâtures de printemps et d'automne sont les communaux ainsi que les prairies de fauche - souvent privées - à l'automne. Ces pâtures se situent dans les limites administratives de chaque village. Enfin, les cultures se trouvent en général au niveau des zones de replats, plus ou moins éloignées des zones habitées.
Les pâturages d'estive correspondent à la zone la plus haute en altitude, accueillant les troupeaux de presque tous les villages durant l'été. Ces parcelles sont presque toutes propriétés de l'État, à qui les éleveurs payent des droits d'usage pour trois ans. Les limites des lots attribués sont revues chaque année. La charge est limitée à cinq petits ruminants ou un bovin par hectare. Il existe une logique spatio-temporelle qui guide les transhumants : les troupeaux pâturent autour du village à partir de mars ou avril selon les éleveurs et la météo. Ils rentrent tous les soirs aux étables. Début juin, lorsque le temps devient plus chaud, ils montent à Rrungaja où ils restent de manière permanente jusqu'à mi-septembre - fin de lactation des brebis -, voire fin novembre pour certains. Les bergers dorment dans des cabanes, souvent temporaires. Les troupeaux sont parqués en extérieur. Durant cette période, le lait est descendu à cheval en bas des estives, puis acheminé jusqu'à la laiterie en véhicules $4 \times 4$. Aux retours des estives, les troupeaux suivent la même logique qu'au printemps avant d'hiverner à l'étable à partir d'octobre-novembre. En général, ce temps de stabulation est de cinq à six mois, rarement quatre.

La zone d'étude compte cinq villages, soit 350 éleveurs sur une surface de $75 \mathrm{~km}^{2}$. Les pâturages sont caractérisés par leur état et leur utilisation par les différents éleveurs-la zone d'étude intègre les villages où se trouvent les acteurs qui utilisent la ressource. Il existe deux types de village autour du 


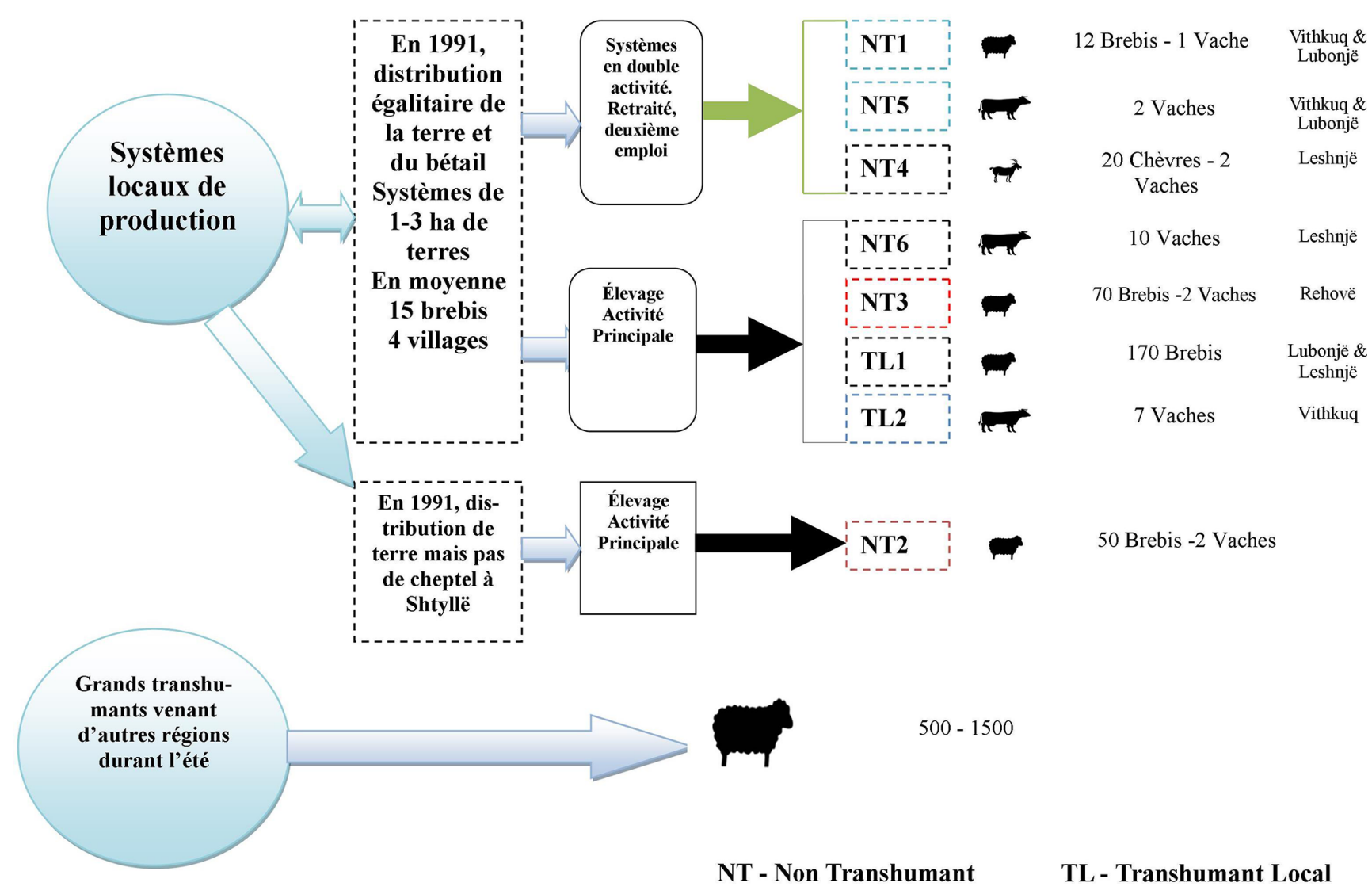

Fig. 2. Coexistence des systèmes d'élevages locaux.

Fig. 2. Coexistence of local livestock systems. (Source: d'après Gontard, 2016; élaboré par les auteurs).

massif: les villages de Vithkuq et Shtyllë se trouvent à proximité de la ressource, et les éleveurs y possèdent certains pâturages, emmènent leurs troupeaux pâturer sur les flancs du massif et, pour certains, effectuent une transhumance estivale dans les alpages; les trois autres villages (Lubonjë, Rehovë et Leshnjë) se trouvent plus loin de la ressource et certains éleveurs effectuent une transhumance estivale sur les pâturages les plus hauts. Par ailleurs, les grands transhumants, éleveurs extérieurs à la région, passent une partie de l'année dans le massif et sont des acteurs importants de l'utilisation et de la gestion de la ressource. Ils sont originaires d'autres régions du pays, surtout du sud, et utilisent chaque année les pâturages privés du massif.

\subsection{Coexistence des systèmes d'élevages locaux: entre survie et spécialisation}

Les systèmes de production actuels sont marqués par les redistributions initiales de la ressource pastorale, mais de nombreux facteurs influencent leur dynamique. Trois critères différencient les systèmes de production:

- Le type de conduite des troupeaux au pâturage l'été:

$\mathrm{NT}=$ Non transhumants ; $\mathrm{TL}=$ Transhumants locaux ;

$\mathrm{GT}=$ Grands transhumants extérieurs.

- La spécialisation (chèvre, brebis, vache).

- La taille du troupeau.
Aujourd'hui, les systèmes d'élevage sont très dépendants de l'accès à la ressource pastorale. La figure 2 schématise la coexistence des systèmes d'élevages locaux et le tableau 1 montre les résultats économiques et les pressions sur la demande en pâturages de chaque système d'élevage.

\subsubsection{Système d'élevage NT1}

Le système d'élevage NT1 est le plus commun de la zone d'étude. Il est caractéristique du village de Vithkuq et comprend des familles de doubles-actifs ou de retraités qui gardent un petit troupeau et un lopin de terre. Sur les 170 éleveurs de Vithkuq, une centaine d'éleveurs correspondent à ce système. Les produits sont majoritairement autoconsommés et le lait vendu est un complément de revenu pour les éleveurs. Les revenus de la double activité (salaires ou retraites) sont importants pour le maintien de ce système d'élevage puisqu'ils comptent pour plus de la moitié des revenus du système d'activité. Ce système est moins exigeant en main-d'œuvre que les systèmes au cheptel plus important, car les éleveurs regroupent leurs troupeaux pour emmener les brebis chacun leur tour au pâturage.

\subsubsection{Système d'élevage NT2}

Le système NT2 est typique du village de Shtyllë, qui est situé à une altitude plus élevée que les autres villages $(1600 \mathrm{~m}$ contre $1200 \mathrm{~m}$ pour Vithkuq). L'hiver y est plus long et les 
Tableau 1. Résultats économiques et pression sur la demande en pâturages de chaque système d'élevage.

Table 1. Economic results and pressure on pasture demand of each livestock system.

\begin{tabular}{|c|c|c|c|c|}
\hline & Taille troupeau & Pâturages & Résultats économiques pour chaque système & Pression sur la demande \\
\hline NT1 & $\begin{array}{l}12 \text { Brebis } \\
1 \text { Vache }\end{array}$ & Communaux & $3440 € / \mathrm{An}$ & Faible \\
\hline NT2 & $\begin{array}{l}50 \text { Brebis } \\
2 \text { Vaches }\end{array}$ & Communaux & $5763 € / \mathrm{An}$ & Moyenne \\
\hline NT4 & $\begin{array}{l}20 \text { Chèvres } \\
2 \text { Vaches }\end{array}$ & Communaux & $3940 € / \mathrm{An}$ & Faible \\
\hline NT5 & 2 Vaches & Communaux & $2661 € / \mathrm{An}$ & Faible \\
\hline TL2 & 7 Vaches & Communaux et Étatiques & $7267 € / \mathrm{An}$ & Moyenne \\
\hline GT1 & 200 Brebis & Privés & $18033 € / \mathrm{An}$ & Haute \\
\hline GT2 & 500 Brebis & Privés & $61167 € / A n$ & Haute \\
\hline
\end{tabular}

(Source: d'après Gontard, 2016; élaboré par les auteurs).

températures plus fraîches, ce qui rend la culture des céréales difficile. La période pendant laquelle les brebis restent à l'étable est plus étendue (six mois contre deux à trois dans les autres villages). Les éleveurs ont quelques hectares de prés de fauche pour la base alimentaire, mais l'achat de maïs dans la plaine de Korçë pèse sur la valeur ajoutée des productions. Ce système est composé d'une cinquantaine de brebis. Au-delà de 60 brebis, le coût de la base alimentaire devient important et la main-d'œuvre manque lors de la fauche. Peu d'éleveurs continuent d'augmenter leur troupeau. En dessous de 40 brebis, il est difficile de faire vivre une famille sans revenus complémentaires dans le système d'activité.

\subsubsection{Système d'élevage NT3}

Ce système est en croissance et pourrait être défini comme un système transitoire entre NT1 et TL1. Il comprend une dizaine de troupeaux, majoritairement à Rehovë, qui sont en augmentation. Les pâturages sans statut autour de Rehovë (sous-bois ou prés de petite taille) ne suffisent plus, surtout au cœur de l'été, au moment où l'herbe commence à sécher (léger déficit hydrique); de nombreux éleveurs envisagent de louer une parcelle de pâturage étatique située plus haut dans le massif. Cependant, ces parcelles, et notamment celles situées le plus près du village, sont celles qui sont les plus demandées par les transhumants de Lubonjë et Leshnjë (TL1).

\subsubsection{Système d'élevage NT4}

Ce système est l'équivalent du système NT1 mais spécialisé en chèvres, et avec les différences suivantes:
(1) concernant les produits, le chevreau est vendu un peu plus cher que l'agneau, le taux de natalité est aussi un peu plus élevé. Le lait est vendu moins cher mais la chèvre en produit presque deux fois plus; (2) concernant l'alimentation, la chèvre peut sortir plus longtemps l'hiver, elle ne reste qu'un mois à l'étable et peut se contenter d'un peu de maïs, voire de branches séchées. En plus, comme dans la plupart du pays, les éleveurs font sécher le foin sur des chevalets en bois appelés «cavaliers » ou «perroquets ». Les pâturages forestiers, qui sont communaux, sont aussi une source importante pour ce système. Peu d'éleveurs pratiquent ce système d'élevage car il est plus difficile d'emmener les chèvres au pâturage. La population est vieillissante et les éleveurs retraités préfèrent garder une vingtaine de brebis (NT1) plutôt que des chèvres. Cependant, quelques élevages de plus de 50 chèvres se maintiennent dans la zone d'étude, avec des éleveurs plus jeunes qui peuvent plus facilement emmener le troupeau au pâturage.

\subsubsection{Système d'élevage NT5}

Ce système est l'équivalent des systèmes NT1 et NT4, mais spécialisé en bovins. Les éleveurs sont aussi double-actifs ou retraités. Les éleveurs s'organisent en troupeaux rassemblés pour éviter d'emmener leurs vaches aux pâturages tous les jours. Comme les systèmes NT1 et NT4, ce système se maintient grâce aux revenus extérieurs (double activité et retraite) et les éleveurs sont plutôt en phase de décapitalisation : les retraités vendent une vache quand ils vieillissent et les double-actifs investissent plutôt dans leur seconde activité - d'autant que depuis 2016, année des 
enquêtes, la construction d'une route reliant Vithkuq à Shtyllë a créé de nombreux emplois.

\subsubsection{Système d'élevage NT6}

Ce système serait l'équivalent du système NT3 mais spécialisé en bovins. Il s'est surtout développé dans les villages avec un accès à la plaine (Leshnjë et Lubonjë), et à Rehovë où l'émigration forte a laissé de nombreuses terres disponibles qui peuvent aussi être cultivées pour de la base alimentaire. Ce système est globalement en croissance, mais l'augmentation du cheptel est conditionnée par la main-d'œuvre, les débouchés pour les produits et l'accès aux pâturages.

\subsubsection{Système d'élevage TL1}

Ce système possédant entre 150 et 200 brebis est principalement présent à Leshnjë et à Lubonjë, où les troupeaux de brebis ont grandi plus vite que dans les autres villages. Plusieurs facteurs peuvent expliquer cette dynamique plus rapide : accès à une plaine et base alimentaire plus facile à obtenir, transferts de l'émigration économique réinvestis dans certains troupeaux à Leshnjë, pas d'autres possibilités que l'élevage à Lubonjë.

\subsubsection{Système d'élevage TL2}

Ce système est principalement composé d'un troupeau rassemblé de quatre éleveurs possédant chacun entre 5 et 10 vaches. À Vithkuq, ce sont surtout des éleveurs du système NT5 qui ont décidé d'augmenter leur troupeau récemment et ont débuté la transhumance. La difficulté de ce système est la quantité de main-d'œuvre nécessaire à son fonctionnement; la transhumance coïncide avec l'époque de fauche, de sarclage du maïs, de récolte de l'avoine et de la luzerne. De nombreux éleveurs préfèrent acheter de la base alimentaire et cela augmente les coûts.

\subsubsection{Système d'élevage GT1}

Ces grands transhumants viennent essentiellement du sudouest de l'Albanie. L'hiver, le climat y est plus doux, il n'y a presque pas de neige et les animaux pâturent sur une période plus longue. C'est un avantage important, les éleveurs passent moins de temps à cultiver de la base alimentaire ou en achètent des quantités plus faibles. En revanche, l'été est plus chaud et sec sur la côte méditerranéenne et les pâturages baissent en qualité. Ces transhumants louent alors les parcelles d'altitude du massif de Rrungaja, encore vertes et de bonne qualité pour les troupeaux. Les troupeaux ont pu augmenter plus vite et la plupart des éleveurs rencontrés font la transhumance sur de longues distances depuis une dizaine d'années. Ce sont des exploitations familiales spécialisées qui engagent au moins un berger pour la période de la transhumance. Les propriétaires du troupeau accompagnent les brebis durant la transhumance (à pied) et restent avec le troupeau dans le massif de Rrungaja.

\subsubsection{Système d'élevage GT2}

Ce sont des exploitations agricoles que l'on pourrait qualifier de capitalistes. Les chefs d'exploitation ont d'autres activités dans leur région d'origine : commerçants, bouchers, entrepreneurs de travaux agricoles, producteurs de maïs, etc. Les troupeaux restent en augmentation tant que l'activité est rentable, et elle l'est pour ces deux élevages car leur région d'origine est l'une des plus touristiques de l'Albanie, ce qui permet de vendre tout l'été leurs produits aux touristes à un prix supérieur à celui du marché. À la différence du système d'élevage GT1, les propriétaires du troupeau ne travaillent pas sur l'exploitation et ne restent pas avec le troupeau à Rrungaja, la transhumance se faisant en camion. Les propriétaires des deux troupeaux du système GT2 engagent des bergers pour s'occuper des brebis. Ils vendent le lait aux laiteries locales, mais leur objectif est la viande fraîche destinée aux villes balnéaires du sud telle que Saranda.

\subsection{Stratégies d'acteurs et évolutions institutionnelles}

À l'exception des éleveurs des systèmes NT1 et NT5, la stratégie dominante des éleveurs de la zone est d'augmenter la production. Les facteurs qui influencent cette augmentation sont: a) l'agrandissement et la redynamisation des troupeaux notamment grâce à l'installation de jeunes de retour d'émigration ; b) la croissance du nombre de petits ruminants adaptés aux conditions géographiques de la zone; c) le prix de la viande en augmentation sur les dernières années. Selon Çili et al. (2013), le coût moyen de la production du lait représente $58 \%$ du prix de vente pour le lait de brebis et $48 \%$ pour le lait de chèvre. Pour la viande, le coût moyen représente respectivement $61 \%$ pour les agneaux et $51 \%$ pour les chevreaux. Malgré ces chiffres apparemment plus avantageux pour la chèvre, celle-ci est en déclin dans la commune et ses zones de pâturage sont délaissées. L'abandon des machines agricoles et les difficultés à réinvestir dans du matériel et des infrastructures sont autant de facteurs augmentant la dépendance des exploitations à une main-d'œuvre jeune et en forme physique (Michaud, 2015) : traite à la main, travail du sol avec des outils rudimentaires, traction animale demandent une force humaine importante. Les éleveurs se sont spécialisés selon leur disponibilité en main-d'œuvre et leur forme physique. Plusieurs éleveurs s'orientent vers la viande bovine qui permet d'accroître leurs recettes annuelles. L'organisation de la filière du lait dans cette zone est contrôlée par les laiteries locales qui sont en position de force pour déterminer le prix de vente du lait. La filière de la viande est totalement informelle. Les éleveurs vendent les animaux aux intermédiaires qui sont souvent des bouchers. Les marges sont meilleures que celle du lait.

Les changements institutionnels de 2016 sur la gestion des ressources pastorales et forestières ont fusionné les pâturages communaux (libre accès et gratuits) et les pâturages étatiques, en «pâturages publics», modifiant les mécanismes de gouvernance. L'objectif principal était d'achever le transfert des pâturages et forêts aux municipalités en favorisant leur capacité à les utiliser durablement. Plus précisément, les pâturages et les forêts communaux sont dorénavant gérés au niveau du district, puisque les municipalités ont été intégrées dans des unités administratives plus larges gérées par les districts. Ces pâturages «publics» sont donnés préférentiellement aux agriculteurs locaux qui doivent maintenant les payer, engendrant des droits exclusifs pour ceux qui les utilisent. 
Tableau 2. Stratégies d'acteurs après les changements institutionnels de la ressource pastorale.

Table 2. Stakeholders' strategies after the institutional changes of pastoral resource.

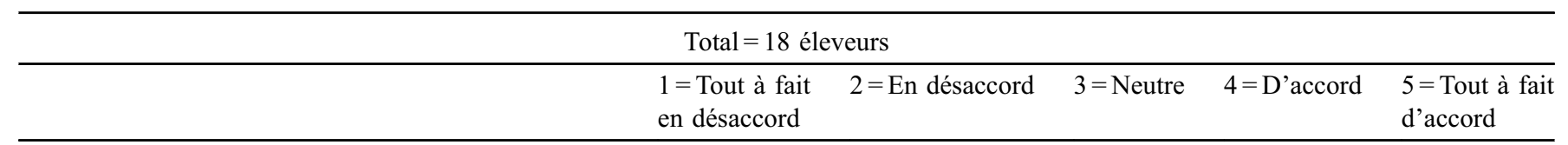

\section{Questions sur la structuration de la filière}

Les pâturages sont une ressource clé pour mieux

valoriser le lait et la viande

Une gestion commune des pâturages peut servir de

$\mathrm{du}$ lait

Une association entre les éleveurs pour le lait va

permettre de mieux vendre le fromage

Une association entre les éleveurs pour la viande va

L'allocation de la ressource pastorale est un des enjeux centraux de la durabilité de nombreuses exploitations du massif. La réforme territoriale, en cours depuis 2016, pose les enjeux de gouvernance de cette ressource. En effet, différents modes de propriété régissaient ces pâturages avant 2016. Le manque de règles bien définies a exercé une pression sur l'accès aux pâturages pour les agriculteurs locaux et, par conséquent, entraîne des problèmes de surpâturage. Dans ces nouvelles conditions, les stratégies commerciales des acteurs peuvent converger ou diverger. Les réponses sur la perception des changements institutionnels de la ressource pastorale montrent un panorama assez diversifié (Tab. 2).

Les éleveurs locaux considèrent les pâturages comme une ressource clé pour mieux valoriser leurs produits, même si les prix actuels du lait et de la viande ne sont pas assez élevés pour payer la location des pâturages après la réforme. Ils voient l'insertion dans une association d'éleveurs comme un moyen pour augmenter leurs volumes de produits pastoraux.

\section{Discussion}

Les résultats économiques amènent les constats suivants. Les systèmes NT1, NT4 et NT5 se maintiennent difficilement, grâce à des sources de revenus extérieurs cruciales. De nombreux agriculteurs sont retraités et décapitalisent en vieillissant. Ces systèmes d'élevage ne sont pas en mesure d'augmenter leur capital et pourraient disparaître progressivement. Pourtant, ces dernières années, de jeunes migrants issus de ces familles ont repris l'activité de leurs parents, investissant une partie de leurs revenus issus de l'immigration dans un plus grand troupeau. 
Les dynamiques de systèmes tels que NT2 et NT3 sont limitées par les besoins de l'alimentation animale en hiver: le système NT2 dans le village de Shtyllë ne peut pas évoluer car l'hiver est trop long. Les agriculteurs ayant des systèmes d'élevage avec plus de 60 brebis doivent être capables de supporter des coûts d'alimentation hivernale importants. La main-d'œuvre nécessaire est le facteur limitant, notamment pour des troupeaux dépassant 70 brebis. Le système d'élevage NT3 génère d'importants problèmes de surpâturage. Les agriculteurs ont du mal à démarrer la transhumance car la pression sur les pâturages publics est déjà forte.

Les systèmes spécialisés NT6 et TL2 sont assez dynamiques. S'ils sont exigeants en main-d'œuvre, les revenus sont comparables à ceux d'autres systèmes comme le NT2 et NT3. Leur dynamique demeure limitée : (1) par la disponibilité en main-d'œuvre, notamment familiale; (2) par les possibilités de sécuriser des pâturages à l'avance. Ce sont ces systèmes qui ont principalement exercé la pression sur les pâturages communaux et publics.

Enfin, les systèmes d'élevage TL1 et GT1 ont des revenus plus élevés et pourraient continuer de s'accroître, tant qu'ils continueront à trouver des pâturages d'État. Les systèmes GT1 et GT2 ont des ressources financières pour louer des pâturages privés, dont les loyers sont en augmentation. Ils exercent une concurrence forte sur les surfaces pastorales de la zone étudiée, en en limitant l'accès aux éleveurs locaux.

Les systèmes d'élevage dont la dynamique est la plus menacée sont les systèmes NT3 et TL1. S'ils ne parviennent pas à obtenir des pâturages d'État, les bêtes doivent rester sur des pâturages d'altitude moyenne, alors que des agriculteurs du système NT3 souhaitent débuter une transhumance estivale afin de continuer à augmenter la taille de leur troupeau. En raison de la pression sur les pâturages d'État, le système NT3 ne pourra vraisemblablement pas évoluer vers le système TL1. Du fait de l'accaparement des surfaces pastorales privées par les grands transhumants externes, l'évolution des systèmes TL1 et GT1 est également affectée. Si la parcelle qu'ils parviennent à louer est à sa charge maximale, ils ne peuvent pas augmenter leur troupeau.

Les pâturages communaux sont la principale ressource pour les agriculteurs locaux qui ont un intérêt commun dans sa bonne gestion. Cependant, certains d'entre eux souhaitent utiliser la ressource à leur guise, avant la formation collective d'un ensemble de règles de gouvernance. La durabilité de la ressource pastorale est menacée en l'absence de modalités de gestion collective et démocratique, et de règles d'usage limitant sa surexploitation (Fournier et al., 2016).

Tous les villages utilisent les pâturages communaux, mais un seul village (Shtyllë) a établi une combinaison de règles locales permettant aux agriculteurs de bénéficier des nouvelles règles d'attribution des pâturages d'État (Bombaj et al., 2018). Les limites des pâturages communaux sont censées être légalement reconnues par les autorités publiques du district, au bénéfice de ceux qui les utilisent. La faiblesse du dispositif est qu'il repose sur la délégation de l'autorité du groupe à un seul membre qui le représente auprès des pouvoirs publics, et qui loue les pâturages communaux au nom de tous. Les membres du groupe peuvent toutefois contrôler et surveiller quotidiennement les conditions de pâturage. Les conditions qui ont favorisé le dispositif de Shtyllë sont l'homogénéité du groupe d'agriculteurs, les liens sociaux étroits qui les unissent, des limites clairement définies pour les pâturages et les menaces exercées par les grands transhumants pour l'utilisation des pâturages en dehors du village.

Ce qui a changé pour les agriculteurs de Shtyllë, c'est l'opportunité d'avoir des droits exclusifs sur les pâturages communaux, créant les conditions initiales pour l'existence d'un bien commun selon la définition d'Ostrom (2009). L'un des principes d'une bonne gestion d'un bien commun est la construction de règles de gouvernance définies collectivement. En fait, les droits exclusifs ont catalysé la nécessité de définir ensemble des règles pour l'usage des pâturages communs. En créant des mécanismes de coordination appropriés, le groupe du village de Shtyllë est au début de la gestion de cette ressource en tant que bien commun.

Dans notre étude, la majorité des exploitations sont de subsistance et le niveau de fragmentation de leurs terres est élevé, ce qui entraîne une faible efficacité de la production. Les résultats montrent des similitudes avec d'autres cas, comme en Grèce (Hadjigeorgiou et al., 2002), où les agriculteurs dépendent avant tout de l'augmentation de la taille du troupeau pour accroître le revenu familial. La différenciation des systèmes d'élevage dépend des revenus disponibles pour louer des terres, élargir le troupeau et acquérir des bâtiments modernes. Par conséquent, les familles ayant de la maind'œuvre disponible s'adaptent plus rapidement aux opportunités des marchés et, comme dans le cas grec, en visant une amélioration de l'efficacité de la main-d'œuvre plutôt que par des investissements en capital (Hadjigeorgiou et al., 2002). Les faibles investissements en capital peuvent aussi s'expliquer par deux raisons: des taux d'intérêt élevés pour les emprunts bancaires et l'absence de garantie des agriculteurs (Marku, 2016). Le capital et la main-d'œuvre sont des éléments clés de la diversité et du développement des systèmes d'élevage, et la distribution initiale des facteurs de production en 1991 a eu un impact à long terme sur les systèmes de production de presque tous les villages.

La perspective de l'intégration européenne amène alors à explorer plusieurs scénarios pour les filières pastorales albanaises, précisant des analyses antérieures effectuées à l'échelle plus large du secteur agricole (Biba et Kerçuku, 2013 ; Bernard et Lerin, 2017 ; Del'homme, 2019) :

- le scénario de la survie d'une économie de microfundia avec de nombreux petits éleveurs (comme NT1, NT2, NT4, NT5) n'est pas à écarter si les soutiens européens et les activités extérieures des ménages complètent les revenus de l'élevage, dans un contexte de demande pour les produits locaux, et malgré des tensions sur l'accès aux pâturages ;

- le scénario d'une modernisation agricole profitant à quelques éleveurs captant les soutiens européens pour investir, payer l'accès aux pâturages et soutenir la concurrence par les coûts (à l'image de GT2, mais aussi de GT1 ou TL1) semble se dessiner, mais il présente des risques important d'exclusion sociale, d'exode rural et de conflits ;

- un troisième scénario pourrait s'appuyer plus clairement sur les communautés locales, dans le cadre d'une politique nationale et européenne de développement rural favorisant des investissements individuels et collectifs, et la gouvernance des pâturages par ces communautés (à l'image du village de Shtyllë). Dans ce cas, les signes 
officiels de qualité, tels que les indications géographiques (IG) (Fournier, 2008), gérés par des organisations locales de producteurs, peuvent signaler aux consommateurs la qualité des produits issus de ces systèmes d'élevage de montagne, et être source de revenus durables pour les producteurs organisés (Barjolle et al., 2007; Barham et Sylvander, 2011 ; Belmin et Casabianca, 2018). Pour cela, les éleveurs ont besoin d'investir dans des installations respectant les normes européennes et dans une organisation commerciale favorisant l'accès aux marchés urbains d'Albanie et progressivement à de nouveaux marchés au sein de l'Union européenne.

\section{Conclusion}

Pays en transition «post-collectiviste », l'Albanie présente des similitudes avec d'autres pays d'Europe centrale et orientale. La réforme agraire de 1991 a posé des problèmes de développement aux agriculteurs situés dans les territoires de montagne. Dans la majorité des cas, les exploitations sont de petite taille et les éleveurs ne peuvent vendre le surplus sur le marché que par des canaux informels, sans pratiques collectives susceptibles de construire un pouvoir de marché. Dans la zone étudiée, l'élevage reste une source de revenu importante. La demande du marché urbain pour les produits pastoraux provenant de cette zone montagneuse, surtout issus des petits ruminants, a connu une forte augmentation ces dernières années. La croissance de cette demande explique l'agrandissement des troupeaux dans le territoire et de nombreux éleveurs voient l'intérêt de produire en grandes quantités pour se développer économiquement. Cela a engendré une demande de plus en plus forte pour des pâturages peu gérés collectivement, provoquant des conflits entre les éleveurs locaux et les grands transhumants venus de l'extérieur, qui viennent y pâturer durant l'été.

Le cas albanais montre que les systèmes locaux d'élevage se sont progressivement adaptés au nouveau contexte politique et économique après la chute du régime communiste en 1991. Le morcellement des terres persiste encore aujourd'hui, empêchant les exploitations de subsistance de capitaliser et de développer leur activité. Les exploitations de subsistance ont tendance à diversifier leur production et leurs sources de revenus. Les terres agricoles fragmentées et la petite taille des exploitations ne permettent pas de réaliser des économies d'échelle.

Dans ce contexte, des initiatives locales semblent prometteuses pour l'avenir. Les changements institutionnels concernant la gouvernance des pâturages communaux montrent que ces derniers sont préférentiellement accordés aux agriculteurs locaux, qui doivent maintenant payer pour les utiliser. Cette réforme a donné aux bénéficiaires autorisés un accès fondé sur l'attribution de «droits exclusifs». En créant des mécanismes de gouvernance adaptés, l'initiative du village de Shtyllë semble efficace pour gérer la ressource commune. La petite taille du groupe et les limites claires des pâturages communaux ont renforcé la capacité d'action collective du groupe. Les conditions d'une telle capacité restent à analyser dans d'autres zones en Albanie. Quoi qu'il en soit, pour gérer durablement les pâturages communs, une adaptation de l'organisation collective locale est nécessaire et les mesures à prendre sont celles qui encouragent les interactions au niveau local. L'articulation entre des politiques de gestion communautaires, publiques et privées doit être mieux harmonisée avec les besoins locaux, pour créer ainsi des conditions favorables pour une possible intégration du pays à l'Union européenne à l'avenir.

\section{Références}

Addison PFE, Rumpff L, Bau SS, Carey JM, Chee YE, Jarrad FC, et al. 2013. Practical solutions for making models indispensable in conservation decision-making. Diversity and Distributions 19 (5-6): 490-502. DOI: 10.1111/ddi.12054.

Afane A, Gagnol L. 2014. Convoitises et conflits entre ressources pastorales et extractives au Nord-Niger. Afrique Contemporaine 1: 53-68. DOI: $10.3917 /$ afco.249.0053.

Andersson K, Ostrom E. 2008. An Analytical Agenda for the Study of Decentralized Resource Regimes. Policy Sciences 41: 71-93. DOI: 10.1007/s11077-007-9055-6.

Barham E, Sylvander B. 2011. Labels of Origin for Food: Local Development, Global Recognition. Wallingford, UK: CABI Publishing, 128 p. DOI: 10.1079/9781845933524.0000.

Barjolle D, Réviron S, Sylvander B. 2007. Création et distribution de valeur économique dans les filières de fromages AOP. Économies et Sociétés, série Systèmes Agroalimentaires 29(9): 1507-1524.

Beesley D. 1985. Changing land use patterns and sheep transhumance in northeastern Sierra Nevada, 1870-1980. In: Forum for the Association of Arid Lands Studies Texas Tech. Univ., Lubbock, pp. 3-8.

Belmin R, Casabianca F. 2018. The key role of Geographical Indications in the governance of "terroir niches". Insights from three Corsican case studies. In: 13rd European IFSA Symposium. Farming Systems: Facing Uncertainties and Enhancing Opportunities, Autriche, Chania, Greece.

Bernard C, Lerin F. 2017. L'Albanie, une agriculture sans dualisme ? In : Paoli J-C, Anthopoulou T, Ben Saad A, Bergeret P, Elloumi M, Napoleone $\mathrm{C}$, Vianey $\mathrm{G}$, eds. La petite exploitation agricole méditerranéenne, une réponse en temps de crise. (Options Méditerranéennes : Série A. Séminaires Méditerranéens, n. 117). Montpellier (France): CIHEAM-IAMM, pp. 19-38. http://om. ciheam.org/option.php?IDOM=1032.

Biba G, Kerçuku H. 2013. L'évolution/stagnation de l'agriculture post-collectiviste en Albanie. Pour 217(1): 77-85. DOI: 10.3917/ pour.217.0077.

Biba G, Pluvinage J. 2006, La pluriactivité dans l'exploitation agricole, transition ou composante durable de l'organisation de la production en Albanie. Cahiers Agricultures 15(6): 535-541. DOI: 10.1684/agr.2006.0026.

Bombaj F, Barjolle D, Anthopoulou T, Michaud G. 2017. Family farming in the Albanian mountainous areas: Local agro pastoral farming systems and market integration perspectives. The Natural Resource Economics Review (Special issue): 41-52.

Bombaj F, Barjolle D, Casabianca F, Anthopoulou T. 2018. Albanian Municipalities facing decentralisation of pastures' management rules. Food Systems - Systèmes Agroalimentaires 3: 31-59.

Bombaj F, Touzard JM, Barjolle D. 2019. Coexistence des systèmes d'élevages d'une zone montagneuse marginalisée en Albanie de sud-est. Entre stratégie d'acteurs et évolutions institutionnelles. In: $13^{e}$ Journées de Recherche en Sciences Sociales, 12-13 décembre, 2019, INRA-SFER-CIRAD, Bordeaux, France, $18 \mathrm{p}$.

Bourbouze A, François M. 2001. Terroir et fromages en Albanie: recomposition des filières laitières dans une économie en transition. Options Méditerranéennes Série B 28: 177-180. 
Çili A, Caca J, Toska K, Kondura K. 2013. Studimi tekniko-ekonomik i fermave blegtorale te bagetive te imeta ne qarqet Korçë, Shkoder, Diber dhe Kukes dhe propozime politikeberje per zhvillim te qendrueshem te tyre. Korçë: QTTB, 76 p.

Cochet H. 2011. Origine et actualité du « Système agraire » : retour sur un concept. Revue Tiers Monde 3(207): 97-114. DOI: 10.3917/ rtm.207.0097.

Cochet H, Devienne S, Dufumier M. 2007. L'agriculture comparée, une discipline de synthèse? Économie rurale. Agricultures, alimentations, territoires 297-298: 99-112. DOI: 10.4000/econo mierurale.2043.

Del'homme B. 2019. L'agriculture dans l'ouest des Balkans: des vicissitudes de l'histoire récente aux choix politiques douteux. Confluences Méditerranée 108(1): 73-85. DOI: 10.3917/ come.108.0073.

Dhimitri E, Aliolli L, Cucllari F, Cini M. 2012. Aspects of the economic development in rural areas of Albania. Case study Korçë region. Journal of Applied Economic Sciences 7: 24-34.

European Commission. 2019. Commission Staff Working Document Albania 2019 Report. Brussels, Belgium, 114 p.

Fournier S. 2008. Les indications géographiques: une voie de pérennisation des processus d'action collective au sein des Systèmes agroalimentaires localisés? Cahiers Agricultures 17 (6): 547-551. DOI: 10.1684/agr.2008.0250.

Fournier S, Vivien D, Bienabe E, Cerdan C, Durand C, Sautier D. 2016. $12^{e}$ Conférence internationale de l'AFD «Communs et développement "1-2 décembre 2016, Paris, 12 p.

Gontard S. 2016. Diagnostic agraire du massif pastoral de Rrungaja. Région de Korçë Sud-Est de l'Albanie. Mise en valeur des pâturages - principale ressources de ces territoires de montagnes par les systèmes d'élevage. Mémoire de Master. Paris, France : AgroParisTech.

Hadjigeorgiou I, Vallerand F, Tsimpoukas K, Zervas G. 2002. The socio-economics of sheep and goat farming in Greece and the implications for future rural development. Options Méditerranéennes Series B 39: 83-93.

Huband S, McCracken DI, Mertens A. 2010. Long and shortdistance transhumant pastoralism in Romania: Past and present drivers of change. Pastoralism: Research, Policy and Practice 1: 55-71.

INSTAT, Institut des Statistiques Albanais. 2019. Statistiques sur l'agriculture et l'élevage en Albanie. http://databaza.instat.gov.al/ pxweb/en/DST/?rxid=98f5bf09-6b91-4d33-a271-c3a2fb90207d [Consulté en ligne 20/11/2020].

Landais E. 1992. Principes de modélisation des systèmes d'élevage. Les Cahiers de la Recherche-Développement 32: 82-95.

Likert R. 1932. A Technique for the Measurement of Attitudes. Archives of Psychology 140: 1-55.

Lutta A, Robinson L, Wasonga O, Ruto E, Sircely J, Nyangito M. 2020. Economic valuation of grazing management practices: discrete choice modeling in pastoral systems of Kenya. Journal of Environmental Planning and Management 63(2): 335-351. DOI: 10.1080/09640568.2019.1584097.

Manoli C, Ickowicz A, Josien E, Dedieu B. 2011. Comment caractériser les relations entre élevage et territoire ? Une revue de la diversité des approches existant dans la littérature. Rencontres Recherches Ruminants 18: 361-368.

Marku E. 2016. Rural Microcredit: Evidences From Albania. European Scientific Journal 12(10): 104-111. DOI: 10.19044/ esj.2016.v12n10p104.

Mavromati J, Gjeta Z, Mavromati E, Kolaneci V, Molla, A. 2011. Production systems and economics of sheep husbandry in Southern Albania. Biotechnology in Animal Husbandry. Belgrade-Zemun: Institute for Animal Husbandry, 27(3): 1167-1176. DOI: 10.2298/ BAH1103167M.

Michaud G. 2015. Étude sociotechnique des systèmes d'élevage dans une commune pastorale du Sud-Est de l'Albanie. Mémoire de Master. Angers, France: ESA.

Müller D, Munroe DK. 2008. Changing rural landscapes in Albania: cropland abandonment and forest clearing in the postsocialist transition. Annals of the Association of American Geographers 98 (4): 855-876. DOI: 10.1080/00045600802262323.

Ostrom E. 2005. Understanding Institutional Diversity. Princeton et Oxford: Princeton University Press.

Ostrom E. 2009. A general framework for analyzing sustainability of social-ecological systems. Science 325(5939): 419-422. DOI: 10.1126/science.1172133.

Citation de l'article : Bombaj F, Barjolle D, Touzard J-M, Casabianca F, Gontard S. 2021. Systèmes de production locaux et gestion des ressources pastorales en Albanie. Entre stratégies d'acteurs et évolutions institutionnelles. Cah. Agric. 30: 6. 